\title{
Synergistic Endo- and Exo-Interactions Between Blueberry Phenolic Compounds, Grape Variety Fractions, Chocolate Covered Strawberries, and Fruit Smoothies
}

\author{
Caleb T. Epps ${ }^{1}$, Benjamin P. Stenquist ${ }^{1}$, Kevin T. Lowder ${ }^{1}$, Bryan C. Blacker ${ }^{1}$, Richard M. Low ${ }^{1}$, Dennis L. \\ Eggett $^{2} \&$ Tory L. Parker ${ }^{1}$ \\ ${ }^{1}$ Department of Nutrition, Dietetics and Food Science, Brigham Young University, United States \\ ${ }^{2}$ Department of Statistics, Brigham Young University, United States \\ Correspondence: Tory L. Parker, Department of Nutrition, Dietetics and Food Science, Brigham Young \\ University, United States. Tel: 1-801-422-6671. E-mail: tory_parker@byu.edu
}

Received: August 19, 2013 Accepted: September 25, 2013 Online Published: September 29, 2013

doi:10.5539/jfr.v2n6p33 URL: http://dx.doi.org/10.5539/jfr.v2n6p33

\begin{abstract}
The presence of phytochemicals in fruits and vegetables is considered to be of nutritional importance in the prevention of chronic diseases, such as cancer and cardiovascular disease. Through overlapping or complementary effects, the complex mixture of phytochemicals in fruits and vegetables provides a better protective effect on health than single phytochemicals. Previous studies have shown that synergistic interactions between antioxidants in food result in a higher antioxidant capacity than individually isolated antioxidants. Further work is needed to explore other potential synergistic interactions between antioxidant mixtures within foods (endo-interactions) and between foods (exo-interactions) commonly eaten together. A series of studies examined potential synergy between various components of blueberries, grapes, chocolate covered strawberries, and fruit smoothies using multiple antioxidant assays (ORAC, TEAC and DPPH). At the ratio found in blueberries, significant synergy, antagonism, and patterns were found for many phenolic compound combinations, though they were dependent on the assay. Significant synergy was found in the combinations of skin and juice as well as skin, juice, and seed across three grape varieties. Significant synergy was found in the combination of strawberry and $88 \%$ cocoa chocolate in fully dipped strawberries. Fruit smoothies made with blueberries (as opposed to strawberries and raspberries) and soymilk (as opposed to water) exhibited significantly higher antioxidant capacity. The 3 assays measured correlated weakly with each other. This work furthers our understanding of the potential value of complex mixtures and foods in the human diet and is the first to report on the combinations and fractions described.
\end{abstract}

Keywords: antioxidants, cardiovascular health, oxidative stress, fruit, interactions

\section{Introduction}

Plants phenolic compounds serve as cell signaling molecules, toxins to invading pests, and antioxidants (Crozier et al., 2006). The phenolic compounds in fruits and vegetables are considered of vital nutritional importance in preventing chronic diseases, such as cancer and cardiovascular disease (Chu et al., 2002a; Chu et al., 2002b; Steinmetz et al., 1996; Van't Veer et al., 2000). The intricate mixture of phytochemicals in fruits and vegetables provides a better protective health effect than single phytochemicals (Eberhardt et al., 2000; Lila \& Raskin, 2005).

Research investigating the components of fruit (Robards et al., 1999; Franke et al., 2004; Harnly et al., 2006) has highlighted phenolic compounds. There is an inconsistency between the antioxidant capacity of an individual phenolic compound and the antioxidant capacity of the whole fruit (Miller \& Rice-Evans, 1997; Zheng \& Wang, 2003); the whole fruit antioxidant capacity is higher. Possible explanations may include unidentified fruit compounds, the summation of many fruit compounds, or various synergistic interactions between phenolic compounds.

Lila and Raskin (2005) discussed additive or synergistic potentiation in terms of endo-interactions, or interactions within a plant that may alter its pharmacological effects, and exo-interactions, which are unrelated plant component and/or drug interactions. Some antioxidant synergy studies through exo-interactions have been 
published. Liao and Yin (2000) demonstrated that combinations of alpha-tocopherol and/or ascorbic acid with catechin, epicatechin, caffeic acid, myricetin, quercetin, gallic acid, and rutin had greater antioxidant activity than any of the individual compounds in an $\mathrm{Fe}^{2+}$-induced lipid oxidation system. The combination of quercetin $3-\beta$-D-glucoside and an apple extract exhibited synergistic anti-proliferative activity in human breast cancer cells (Yang \& Liu, 2009). The combination of acerola cherry extracts and alfalfa and soy phytoestrogen extracts worked to inhibit LDL oxidation synergistically in vitro (Hwang et al., 2001). Parker et al. (2010) found synergistic interactions between mixtures of rutin, $p$-coumaric acid, abscisic acid, ascorbic acid, and a sugar mixture using oxygen radical absorbance capacity (ORAC) and electron paramagnetic resonance (EPR). Hidalgo et al. (2010) discussed synergy between mixtures of two phenolic compounds, finding synergy in many of the combinations. Antioxidant synergism occurs in a variety of compounds and extracts.

Endo-interactions within a specific food have not been as widely studied. Seeram et al. (2004) discovered synergy among cranberry phytochemicals against malignant cell lines. Su et al. (1987) found a synergist in Osbeckia chinensis. Kayano et al. (2002) found a different synergist in prunes (Prunus domestica). Our laboratory published two in vitro studies (Freeman et al., 2010; Reber et al., 2011) and a small human trial (Snyder et al., 2011) that suggest endo-interactions within a fruit are primary contributors to their health benefits. The ratio of antioxidants in fruit plays a significant role in synergy.

The objective of this paper was to explore various endo- and exo-interactions at the chemical, fruit fraction and whole food level in a series of studies. It was hypothesized that synergism could be demonstrated in all three areas. This could help explain the antioxidant capacity difference between whole fruit and individual components, increase our understanding of how fruit fractions interact, and clarify the potential benefits of consuming certain foods together.

\section{Materials and Methods}

\subsection{Chemicals}

Trolox, potassium persulfate, quercetin hydrate, perchloric acid, and Folin-Ciocalteu reagent were purchased through Fisher Scientific Inc. (Waltham, MA, U.S.A.). (+)-Catechin hydrate, chlorogenic acid, myricetin, malvidin chloride, fluorescein, 2,2'-azino-bis(3-ethylbenzothiazoline-6-sulfonic acid) diammonium salt (ABTS), 2,2 diphenyl-1-picrylhydrazyl (DPPH), acetone, $p$-coumaric acid, (-)-epicatechin, gallic acid, dithiothreitol (DTT), meta-phosphoric acid (MPA), cupric sulfate pentahydrate and pelargonidin chloride were purchased from Sigma-Aldrich (St. Louis, MO, U.S.A.). 2,2'-Azobis(2-methylpropionamidine) dihydrochloride (AAPH) was purchased from Wako Chemicals U.S.A. Inc. (Richmond, VA, U.S.A.). Dimethylformamide (DMF, silylation grade) and N,O-bis(trimethylsilyl) trifluoroacetamide with 1\% trimethylchlorosilane (BSTFA w/ 1\% TMCS) were purchased from Pierce (Rockford, IL, U.S.A).

\subsection{Blueberry Study}

\subsubsection{Chemical Preparation}

The five most concentrated phenolic compounds were chosen and studied at the following ratios: catechin, 7 ; chlorogenic acid, 5; malvidin, 13; quercetin, 3; and myricetin, 1 (USDA Flavonoid Database, 2007; Zheng \& Wang, 2003; Wang et al., 2009). For the TEAC and DPPH assays, all compounds were weighed out, dissolved and diluted in ethanol, and stored at $-80^{\circ} \mathrm{C}$. For ORAC, the compounds were dissolved in methanol, then diluted (7:3 acetone:water), and stored $\left(-80^{\circ} \mathrm{C}\right)$. Phenolics were brought to room temperature (RT), vortexed, and then mixed with minimal indirect light.

\subsubsection{Mixtures and Extraction Preparation}

All possible combinations of 2, 3, and 4 compounds were mixed at the above ratios on the day of their assay. Mixtures were then further diluted to fit a standard curve (ORAC), or give a linear relationship on the concentration-inhibition plot (TEAC and DPPH).

\subsubsection{Oxygen Radical Absorbance Capacity (ORAC)}

The ORAC assay was performed according to Davalos et al. (2004) with some modifications. Briefly, fluorescein, Trolox, and AAPH were diluted to $117.2 \mathrm{nM}$ (phosphate buffer), $80 \mu \mathrm{M}$ (7:3 acetone:water), and $40 \mathrm{mM}$ (phosphate buffer) respectively, final concentration. Fluorescein and AAPH were transferred to all wells of 96-well plates via a Precision Micropipettor (BioTek Instruments, Inc., Winooski, VT, U.S.A.). The standard curve used four concentrations of Trolox $(10 \mu \mathrm{M}, 20 \mu \mathrm{M}, 40 \mu \mathrm{M}, 80 \mu \mathrm{M})$ and the outer ring of wells were filled, but not used, to maintain temperature uniformity. Fluorescence of all wells was measured at 485/20 nm excitation and 528/20 nm emission every minute for 120 minutes in a BioTek Synergy 2 fluorescence plate 
reader (BioTek Instruments, Inc., Winooski, VT, U.S.A.) set at $37{ }^{\circ} \mathrm{C}$ after pre-warming. ORAC values were expressed as Trolox Equivalents (TE) per mmol.

\subsubsection{Trolox Equivalent Antioxidant Capacity (TEAC)}

The TEAC assay was performed according to Re et al. (1999) with slight modifications. Trolox was diluted to $400 \mu \mathrm{M}$ in ethanol. ABTS was dissolved in double distilled (dd) water to a $7 \mathrm{mM}$ concentration. ABTS radical cation $\left(\mathrm{ABTS}^{\circ+}\right)$ was prepared. Diluted $\mathrm{ABTS}^{\circ+}$ solution $(180 \mu \mathrm{l})$ was added to $20 \mu \mathrm{l}$ of antioxidant or Trolox standards (final concentration 5-40 $\mu \mathrm{M}$ ) in a 96-well plate via the Precision Micropipettor. The absorbance reading $(734 \mathrm{~nm})$ was taken at room temperature exactly $30 \mathrm{~min}$ after initial mixing using the Biotek Synergy 2 plate reader. To calculate the TEAC, the slope of percentage inhibition of absorbance vs. concentration is divided by the slope of the plot for Trolox. This gives the TEAC $(\mathrm{mM})$ at $30 \mathrm{~min}$.

\subsubsection{2,2-diphenyl-1-picrylhydrazyl (DPPH)}

Trolox was diluted to $1.5 \mathrm{mM}$ in ethanol. DPPH was dissolved in ethanol to $0.1 \mathrm{mM}$. Diluted DPPH solution $(200 \mu \mathrm{l})$ was added to $50 \mu \mathrm{l}$ of antioxidant or Trolox standards (final concentration 7.2, 15, 30 $\mu \mathrm{M}$ ) in a 96-well plate via the Precision Micropipettor. The absorbance reading $(517 \mathrm{~nm})$ was taken at room temperature exactly 40 min after initial mixing using the Biotek Synergy 2 plate reader. To calculate the DPPH value, the slope of percentage inhibition of absorbance vs. concentration is divided by the slope of the plot for Trolox. This gives the DPPH value expressed as Trolox Equivalents (TE) per mmol.

\subsection{Grape Study}

\subsubsection{Component Separation}

Black Ribier, Red Globe, and Sugraone varieties of grape were purchased locally (Chilean origin). For the whole grape extract, 10 grapes were weighed and blended. Another 10 grapes were weighed to prepare the skin, seed, pulp, and juice components. Each grape was skinned using a spatula, weighed and blended. The seeds, including the stem connector, were collected and weighed, crushed, and mixed with dd water. The remaining pulp and juice were milled three times. The juice was collected and the pulp was scraped back into the mill, and then collected after the final milling. The juice was weighed. The pulp was weighed and then blended with dd water. All samples were stored at $-80^{\circ} \mathrm{C}$.

\subsubsection{Extract Preparation}

Samples were thawed at $26{ }^{\circ} \mathrm{C}$, then vortexed. A sample was weighed. A mixture of acetone:water:acidic acid (AWA, 70:29.5:0.5, $200 \mu \mathrm{l}$ ) was added, then vortexed for 30 seconds, sonicated (Fischer Scientific FS60D, Pittsburg, PA, U.S.A.) at $37{ }^{\circ} \mathrm{C}(5 \mathrm{~min}, 60 \mathrm{degas} /$ minute), and centrifuged (11,000 x g, $1 \mathrm{~min})$. The supernatant was transferred. This was repeated twice more ( $20,000 \mathrm{x} \mathrm{g}, 2 \mathrm{~min}$, after third extraction). Combined supernatants were vortexed, centrifuged $(10,000 \mathrm{x} \mathrm{g}, 30 \mathrm{sec})$ and diluted as necessary.

\subsubsection{Combination Preparation}

All possible combinations of grape components were then prepared. Components were combined based on the percentage mass of that component found in a grape. All combinations were then stored at $-80^{\circ} \mathrm{C}$.

\subsubsection{ORAC Assay}

The ORAC assay was performed according to the blueberry study, except that AWA was used.

\subsubsection{TEAC Assay}

The TEAC assay was performed according to the blueberry study except Trolox was diluted in AWA.

\subsection{Chocolate Covered Strawberries Study}

\subsubsection{Preparation}

Strawberries (California origin) and 54\% cocoa and $88 \%$ cocoa chocolate bars were purchased locally. Strawberries were separated into four groups of ten and weighed. Each group of ten strawberries was either fully-dipped or half-dipped in $54 \%$ or $88 \%$ cocoa chocolate melted at $55-60{ }^{\circ} \mathrm{C}$. Following dipping, each strawberry was dripped $(3 \mathrm{sec})$, frozen $\left(-80^{\circ} \mathrm{C}, 5 \mathrm{~min}\right)$, and weighed. Average ratios of $\mathrm{g}$ chocolate added to $\mathrm{g}$ strawberry were determined. Strawberries were pureed and chocolate was aliquoted separately for extraction.

\subsubsection{Lipophilic Extraction}

Samples were thawed (RT) and extracted with hexane three times via shaking, vortexed (30 sec, $3000 \mathrm{rpm})$, sonicated $\left(5 \mathrm{~min}, 37^{\circ} \mathrm{C}\right.$, interrupted once to shake) and centrifuged (60 sec, 10,000 x g) (Davalos et al., 2004; Prior et al., 2003; Wu et al., 2004). On the fourth and final extraction, samples were sonicated (10 min, with 
shaking) and centrifuged ( $2 \mathrm{~min}, 20,000 \mathrm{xg}$ ). Any remaining liquid in the pellets was evaporated under gentle $\mathrm{N}_{2}$ gas.

\subsubsection{Hydrophilic Extraction}

Hydrophilic extractions were performed as above, except AWA was used in place of hexane. Chocolate samples required eight AWA extractions; strawberry sample required three. It was assumed that all phenolic compounds were extracted when the supernatant was colorless.

\subsubsection{Lipophilic ORAC $\mathrm{LP}_{\text {Assay }}$}

All steps of the $\mathrm{ORAC}_{\mathrm{LP}}$ were identical to those described above except hexane was evaporated and Trolox and all lipophilic extracts were re-dissolved in 100\% acetone.

\subsubsection{Hydrophilic ORAC $\mathrm{HP}_{\mathrm{H}}$ Assay}

The ORAC $_{\mathrm{HP}}$ assay was performed as in the blueberry study, except AWA was used in place of AW. Strawberry and chocolate AWA extracts were combined to represent the $88 \%$ cocoa fully dipped average weight ratio of $g$ chocolate to g strawberry. All combined extractions were diluted with AWA.

\subsection{Fruit Smoothies Study}

\subsubsection{Smoothie Preparation}

Fruit and frozen yogurt (Breyers brand, Original) were purchased pre-frozen locally, and then stored $\left(-20{ }^{\circ} \mathrm{C}\right)$ (except soymilk (Silk brand, original flavor) was refrigerated). The following smoothies were prepared: S1-Fruit Combination + Milk (360 ml Soymilk, $120 \mathrm{~g}$ frozen yogurt and $60 \mathrm{~g}$ ice (milk blend), $60 \mathrm{~g}$ each of blueberries, strawberries, and raspberries (fruit blend)), S2- Strawberries + Milk (milk blend and $180 \mathrm{~g}$ strawberries), S3Blueberries + Milk (milk blend and $180 \mathrm{~g}$ blueberries), S4- Raspberries + Milk (milk blend and $180 \mathrm{~g}$ raspberries), S5- Fruit Combination + Water $(360 \mathrm{ml}$ dd water, $120 \mathrm{~g}$ frozen yogurt, $60 \mathrm{~g}$ ice and fruit blend), C1Milk + Water (360 ml soymilk, $300 \mathrm{ml} \mathrm{dd}$ water, and $60 \mathrm{~g}$ ice), C2- Milk + Frozen Yogurt + Water (360 ml soymilk, $120 \mathrm{~g}$ frozen yogurt, $180 \mathrm{ml}$ dd water, and $60 \mathrm{~g}$ ice), C3- Water + Frozen Yogurt (120 g frozen yogurt, $540 \mathrm{ml}$ dd water, and $60 \mathrm{~g}$ ice).

Ingredients were weighed, mixed (Waring blender, high, $2.5 \mathrm{~min}$ ), then sampled every 15 minutes for 45 minutes and stored $\left(-80^{\circ} \mathrm{C}\right)$. Colorimetric analysis was performed before freezing.

\subsubsection{Total Phenolics}

The total phenolics (TP) assay was performed according to Swain and Hillis (1959) and Serafini et al. (1998). Each smoothie was treated with an acid and base treatment $(\mathrm{HCl}, \mathrm{NaOH}$, and $m$-phosphoric acid) to liberate phenolic compounds, followed by vortex, sonication $\left(37^{\circ} \mathrm{C}, 5 \mathrm{~min}\right)$, centrifugation $(11,000 \mathrm{x} \mathrm{g}, 1 \mathrm{~min})$, extracted with AWA per the chocolate covered strawberries study, and the remaining steps of the Folin-Ciocalteu reaction were performed (final incubation, $25 \mathrm{~min}, 45^{\circ} \mathrm{C}$ ). Absorbance was determined in triplicate (Biotek Synergy 2) at $765 \mathrm{~nm}$ with a gallic acid standard.

\subsubsection{Colorimetric Assay}

Color was measured immediately after collection (Hunterlab Colorflex spectrophotometer, Hunter Associates Laboratory, Inc., Reston, VA, U.S.A). L*a*b* values were measured in triplicate according to instrument instructions.

\subsubsection{Oxygen Radical Absorbance Capacity (ORAC)}

The ORAC assay was performed according to the blueberry study, except that AWA was used.

\section{Statistics}

\subsection{Blueberry Study}

For combinations of two, a difference was calculated by subtracting the sum of the average ORAC, TEAC or DPPH values for the individual compounds from the resulting average value of the combination of both compounds (Equation 1):

$$
\text { Difference }=(\text { combination } a b)-(\text { individual } a+\text { individual } b) \text {. }
$$

Likewise, for combinations of 3 and 4 , the difference was calculated by subtracting the average of the individual 3 or 4 compounds from the combination (Equations 2 and 3).

$$
\text { Difference }=(\text { combination } a b c)-(a+b+c) \text {, }
$$




$$
\text { Difference }=(\text { combination } \mathrm{abcd})-(\mathrm{a}+\mathrm{b}+\mathrm{c}+\mathrm{d}),
$$

Presenting the results in this manner allowed us to easily distinguish those combinations that were at minimum additive, using Fisher's least significant difference (LSD) analysis in the SAS statistical package (version 9.3, SAS Institute Inc., Cary, NC, U.S.A.). Additionally, for combinations of 3 and 4, a difference was calculated by subtracting the sum of the average ORAC values for the combination of 2 or 3, plus 1 individual, from the resulting average ORAC value of the combination of all 3 or 4 compounds (Equations 4 and 5).

$$
\begin{gathered}
\text { Difference }=(\text { combination } a b c)-(\text { combination } a b+\text { individual } c), \\
\text { Difference }=(\text { combination } a b c d)-(\text { combination } a b c+d),
\end{gathered}
$$

SAS was used to determine significance of combinations using estimate statistics, which take into account error terms when data are combined. The above described differences were compared through an ANOVA of the individual and combination results, and forming the differences as post hoc tests to determine the effect of combining the individual compounds and combinations. Presenting the results in this manner allowed us to determine which compounds contributed most to a combination.

\subsection{Grape Study}

Statistical analysis is the same as the blueberry study, except that the analysis was calculated based on grape fraction values.

\subsection{Chocolate Covered Strawberries Study}

Statistical analysis is the same as the blueberry study, except a combination of 5 was analyzed using all possible combinations.

\subsection{Fruit Smoothies Study}

Two analyses were carried out. The first ignored time and looked at all combinations of smoothie comparisons in an ANOVA. This was necessary because the control (C1-C3) samples were not measured over time. The initial ANOVA was followed by post hoc tests of pairwise differences. The second analysis was a two way ANOVA with S1-S5 and time $(0,15,30$, and 45 minutes). This was followed by post hoc pairwise tests between S1-S5 and then time.

\section{Results and Discussion}

\subsection{Blueberry Study}

\subsubsection{ORAC}

Only statistically significant synergistic interactions are included in Figure 1. All of the combinations that were synergistic included catechin or malvidin, suggesting they played an important role in determining the synergy of a combination. Combinations that had both catechin and malvidin were more than twice as often synergistic (31.4\%) compared to those with only one of the two $(12.4 \%)$, suggesting their synergy is an important interaction. 


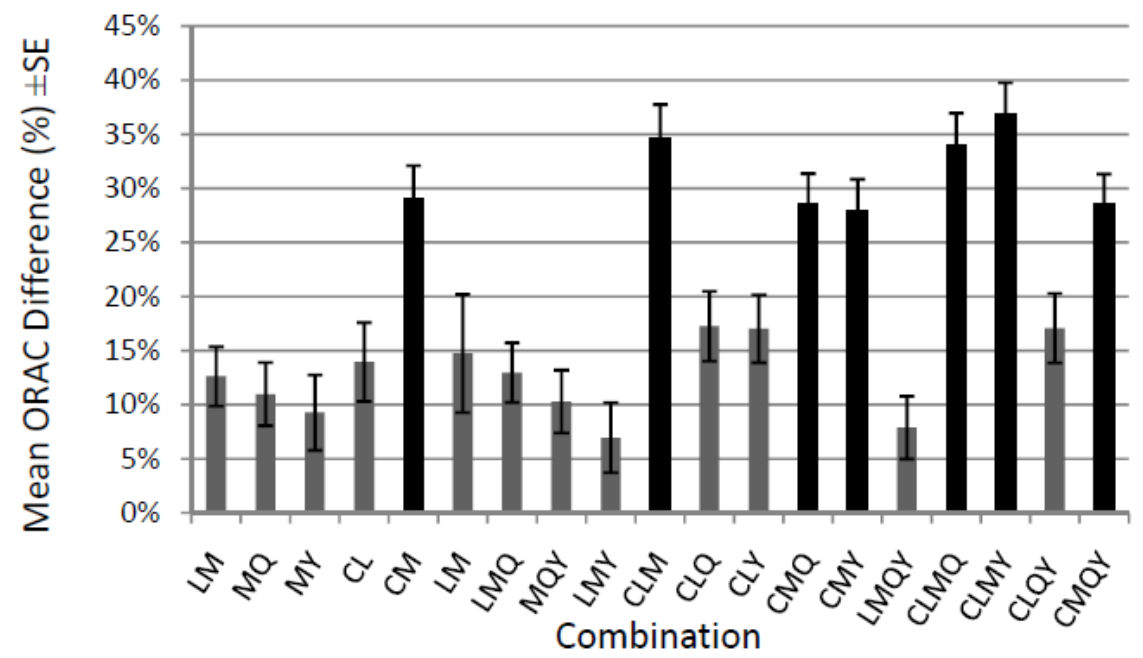

Figure 1. Oxygen Radical Absorbance Capacity (ORAC) differences for combinations minus the individual compounds in the combination (Equation 1 to Equation 3$)$. Only statistically significant $(\mathrm{p}<0.05)$ combinations are shown $(\mathrm{n}=6)$. $\mathrm{C}=$ catechin; $\mathrm{L}=$ chlorogenic acid; $\mathrm{M}=$ malvidin; $\mathrm{Q}=$ quercetin; $\mathrm{Y}=$ myricetin. $\mathrm{LM}$ indicates the ORAC of the mixture of $\mathrm{L}$ and $\mathrm{M}$ minus the ORAC of $\mathrm{L}$ and the ORAC of M; likewise for the other combinations. The difference was then divided by the average value of the combination, giving a percentage. Gray bars contain catechin or malvidin; black bars contain both catechin and malvidin

There appeared to be a positive correlation with the number of compounds in a given mixture with its $\%$ synergy. The average synergy of combinations of 2 compounds was $15.1 \%$, of 3 compounds was $19.5 \%$, and of 4 compounds was $24.9 \%$. As the number of compounds increased, the more likely it was that any given combination would have both catechin and malvidin in it.

When looking at all combinations that contained a particular compound, catechin had the highest \% synergy. Combinations with catechin had an average \% synergy of $25.9 \%$, whereas the average synergy of combinations not containing catechin was $19.0 \%$.

Reber et al. (2011) studied the synergistic effect of 7 phenolic compounds, including catechin, commonly found in strawberries. Their results provide both supporting and refuting evidence. In Reber et al., when catechin was combined with $p$-coumaric acid there was significant synergy. We found a similar result in the catechin+chlorogenic acid combination. However, in a combination of three, catechin $+p$-coumaric acid+pelargonidin, an antagonistic effect was observed by Reber et al. A possible explanation is that the attempt by catechin to donate its electrons to $p$-coumaric acid (synergistic effect) is disrupted by the lack of a catechol group on the pelargonidin. This decreased catechin's effectiveness and resulted in antagonism. We found the opposite with the combination of catechin+chlorogenic acid+malvidin. Structural differences or the ratio of the compounds to each other may account for the differences. Hidalgo et al. (2009) studied the effect of donating electrons from eugenol derivatives; their results support our findings. They found that two eugenol derivatives displayed higher ORAC values due to their ability to stabilize the radical in the ortho $(o)$ and para $(p)$ positions of an aromatic ring. Three other derivatives had substituents that disrupted the stabilizing effect of the conjugated double bonds.

\subsubsection{TEAC}

For the TEAC assay, there were 2 combinations of 2 antioxidants that had statistically significant antagonistic interactions, and 3 combinations of 3 antioxidants that had statistically significant synergistic interactions (Figure 2). The myricetin+malvidin combination showed synergy when a $3^{\text {rd }}$ compound was added, suggesting myricetin plays a key role in determining the strength of the interaction. Four out of the 5 combinations with significant interactions contained myricetin.

For the synergistic combinations of 3 in Figure 2, no individual compound or pair of compounds was responsible for the synergy. All corresponding possible $2+1$ calculations for synergistic combinations were also synergistic 
(CLQ and CMY). Thus there was no indication that any compound was more important than another. In addition, none of the combinations of 4 , per eq. 3 , were significant.

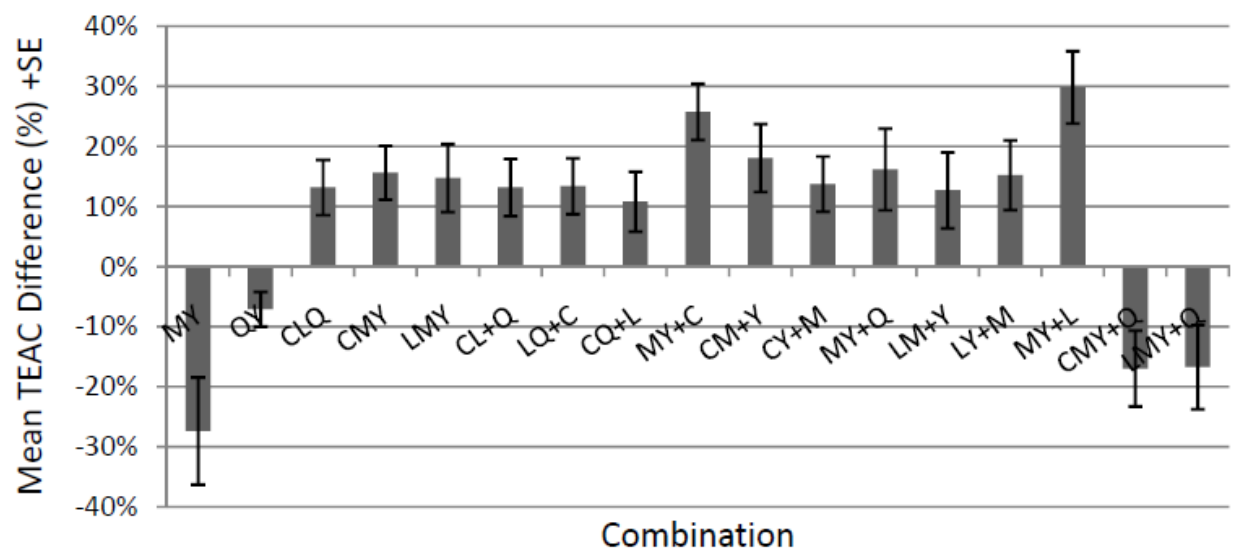

Figure 2. Trolox Equivalence Antioxidant Capacity (TEAC) for combinations minus the individual compounds in the combination (Equation 1 to Equation 3, no plus sign) and combinations of 3 phenolic compounds minus the sum of the $2+1$ data (Equation 4, plus sign) or $3+1$ data (Equation 5, plus sign). Analysis of the data by adding one compound at a time elucidates patterns and makes it possible to determine which compound interactions are most influential. Only statistically significant $(\mathrm{p}<0.05)$ combinations are shown $(\mathrm{n}=3)$. $\mathrm{C}=$ catechin; $\mathrm{L}=$ chlorogenic acid; $\mathrm{M}=$ malvidin; $\mathrm{Q}=$ quercetin; $\mathrm{Y}=$ myricetin. The difference was then divided by the average value of the combination, giving a percentage

Combinations of 3 that were synergistic (CLQ, CMY, LMY) showed antagonism if quercetin was added as the fourth compound (data not shown). Overall, adding a $3^{\text {rd }}$ compound had a synergistic effect on the mixture, whereas adding a 4th compound had an antagonistic effect. When a $3^{\text {rd }}$ compound was added to MY, a higher level of synergy $(23.9 \%)$ was found than with other mixtures to which a $3^{\text {rd }}$ compound was added (13.9\%). Both significant $3+1$ calculations yielded antagonistic effects, contained MY, and Q was added.

\subsubsection{DPPH}

The DPPH assay showed primarily antagonistic interactions (Figure 3). All of the combinations that had statistically significant antagonism included chlorogenic acid in them. There was only one synergistic combination. Adding chlorogenic acid or malvidin to 2 or 3 other compounds had an antagonistic effect. Six out of seven of the antagonistic interactions found when adding a single compound to a combination came from the addition of either chlorogenic acid or malvidin. Wang et al. (2011) found $25 \%$ of their tested whole food combinations exhibited an antagonistic DPPH interaction. The synergistic effect of a mixture of two foods within the same category was generally lower than a mixture of two foods from across food categories. This is consistent with our DPPH results as two foods within the same food category are most similar to our data.

\subsubsection{Correlation Between ORAC, TEAC, and DPPH Assays}

Only two significant correlations occurred between the different assays. When measuring the synergy of antioxidant combinations, we found a significant negative correlation between the ORAC and TEAC assays ( $\mathrm{r}=$ $-0.296, \mathrm{p}<0.05)$. When measuring the individual compound antioxidant capacity, we found a significant negative correlation between ORAC and DPPH $(r=-0.308, \mathrm{p}<0.05)$. Only three combinations showed synergy across multiple assays. Catechin+chlorogenic acid+quercetin, catechin+malvidin+myricetin, and chlorogenic acid+malvidin+myricetin were significantly synergistic in both the TEAC and ORAC assays. None of the compounds that had synergy overlapped with the DPPH assay. 


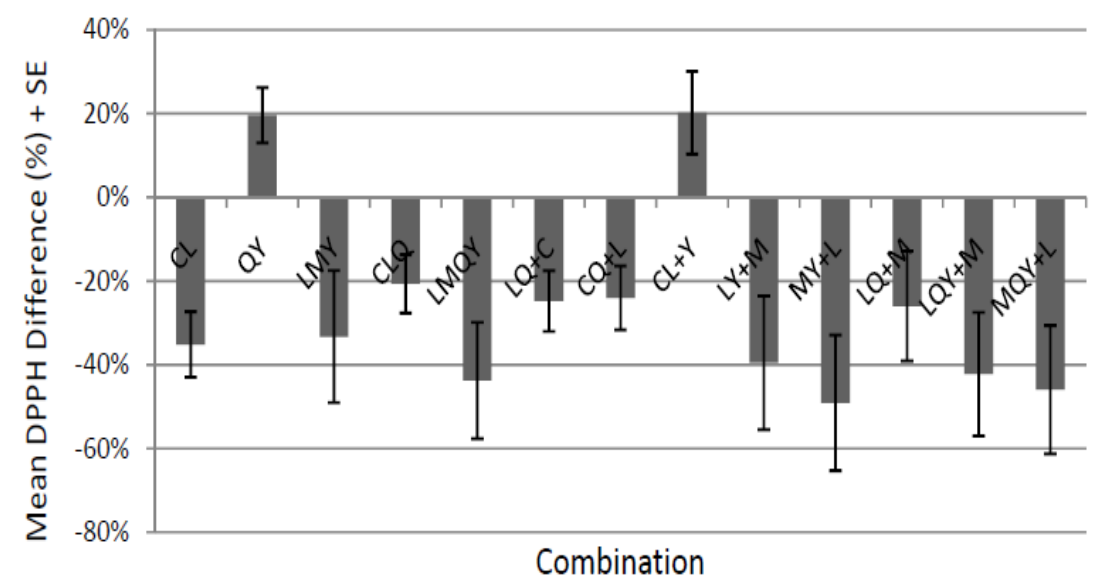

Figure 3. DPPH antioxidant capacities for combinations minus the individual compounds in the combination (Eq. 1 to Equation 3, no plus sign) and combinations of 3 phenolic compounds minus the sum of the $2+1$ data (Equation. 4, plus sign) or $3+1$ data (Equation 5, plus sign). Analysis of the data in this plus-one way elucidates patterns and makes it possible to determine which compound interactions are most influential. Only statistically significant $(\mathrm{p}<0.05)$ combinations are shown $(\mathrm{n}=3) . \mathrm{C}=$ catechin; $\mathrm{L}=$ chlorogenic acid; $\mathrm{M}=$ malvidin; $\mathrm{Q}=$ quercetin; $\mathrm{Y}=$ myricetin. The difference was then divided by the average value of the combination, giving a percentage

The DPPH and TEAC assays used electron-deficient radicals. However, the correlations between them was not high $\left(r^{2}=0.47\right)$, which is similar to the result reported by Tabart et al. (2009) $\left(r^{2}=0.36\right)$. Tabart et al. compared the antioxidant capacities (exo-interactions) of several compounds using ORAC, TEAC, and DPPH. After testing 25 compounds, they found little agreement across the varying assays in antioxidant values. Only a few compounds such as myricetin and gallocatechin gave similar results across assays. In the DPPH assay, no antioxidant capacity was observed in quercetin, kaempferol, cyanidin, rutin, gallic acid, cyanidin-3-O-glucoside, and cyanidin-3-O-rutinoside. However, all of these flavonoids exhibited greater activities than Trolox in the TEAC assay. The TEAC assay in general was higher for more compounds than DPPH (68\% compared to 52\%). This trend is similar to the findings in this paper ( $76 \%$ compared to $15 \%)$, though our correlations were based on synergy values rather than individual compound values.

In contrast, Awika et al. (2003) reported a high correlation between TEAC and DPPH with ORAC $\left(\mathrm{R}^{2}=0.99\right.$ and $\mathrm{R}^{2}=0.97$, respectively) while measuring whole foods (i.e. bran bread, bran cookie, etc.). In our measurements of whole food synergy (see below), we did not see this same trend.

Wang et al. (2011) studied the antioxidant capacities of mixtures of legumes, vegetables, and fruits, looking for synergistic exo-interactions. Their results showed that the DPPH assay is more likely to find additive or antagonistic interactions, whereas the ORAC assay is more likely to find additive or synergistic interactions. Our results confirmed this observation.

\subsection{Grape Study}

\subsubsection{ORAC}

Individual ORAC values were obtained for each of the five components (whole, juice, seed, skin, and pulp) across three different grape varieties (Vitis vinifera, cultivars Black Ribier, Red Globe, and Sugraone). When comparing the cultivars to each other (Table 1), the Black Ribier cultivar had the highest $(\mathrm{p}<0.05)$ ORAC value for the whole grape, juice, and skin. The Red Globe variety had the highest $(p<0.05)$ values for seed, and Sugraone had the highest $(\mathrm{p}<0.05)$ value for pulp. A possible explanation for the Black Ribier showing greater values in whole, juice, and skin is the greater concentration of anthocyanin antioxidant compounds found in darker colored skins (Gao \& Cahoon, 1994). The whole grape also had a higher antioxidant capacity because the skin was included. Black Ribier may have had more soluble antioxidants diffuse into the juice or it produced more of them. Conversely, the seed and pulp components may be more influenced by cultivar. This is consistent with Lutz et al. (2011) who found that blue grape juice (Autumn Royal, Ribier) phenolic content and antioxidant capacity was higher than red grape juice (Red Globe, Crimson Seedless). They also found that other 
health-promoting phenolics are abundant mainly in the skin fraction of blue grapes. The juice examined in the present study was extracted directly by milling the grape with no other processing, unlike Lutz et al.

Table 1. ORAC and TEAC values for each component of each grape variety ${ }^{1}$

\begin{tabular}{lllllll}
\hline \multicolumn{4}{c}{ ORAC $(\boldsymbol{\mu m o l ~ T E} / \mathbf{g})$} & \multicolumn{3}{l}{ TEAC(mM) } \\
\hline Component & Black Ribier & Red Globe & Sugraone & Black Ribier & Red Globe & Sugraone \\
Whole & $22.3 \pm 2.0 \mathrm{a}$ & $6.40 \pm 2.0 \mathrm{~b}$ & $7.50 \pm 2.0 \mathrm{~b}$ & $1.26 \pm 0.2 \mathrm{a}$ & $0.516 \pm 0.2 \mathrm{a}$ & $0.653 \pm 0.2 \mathrm{a}$ \\
Juice & $3.34 \pm 0.2 \mathrm{a}$ & $1.43 \pm 0.2 \mathrm{~b}$ & $1.72 \pm 0.2 \mathrm{~b}$ & $0.370 \pm 0.1 \mathrm{a}$ & $0.09 \pm 0.1 \mathrm{~b}$ & $0.09 \pm 0.1 \mathrm{~b}$ \\
Seed & $75.9 \pm 7.0 \mathrm{a}$ & $114 \pm 7.0 \mathrm{~b}$ & $79.9 \pm 7.0 \mathrm{a}$ & $0.396 \pm 0.03 \mathrm{a}$ & $1.08 \pm 0.03 \mathrm{~b}$ & $0.522 \pm 0.03 \mathrm{a}$ \\
Skin & $93.3 \pm 5.0 \mathrm{a}$ & $40.8 \pm 5.0 \mathrm{~b}$ & $36.0 \pm 5.0 \mathrm{~b}$ & $0.89 \pm 0.1 \mathrm{a}$ & $0.99 \pm 0.1 \mathrm{a}$ & $0.59 \pm 0.1 \mathrm{a}$ \\
Pulp & $3.15 \pm 0.3 \mathrm{a}$ & $2.92 \pm 0.3 \mathrm{a}$ & $4.30 \pm 0.3 \mathrm{~b}$ & $0.08 \pm 0.03 \mathrm{a}$ & $0.04 \pm 0.03 \mathrm{a}$ & $0.11 \pm 0.03 \mathrm{a}$ \\
\hline
\end{tabular}

${ }^{1}$ Grape components that do not share a lowercase letter are statistically different $(\mathrm{P}<0.05)$ within each component row and assay. $n=3$.

Synergy was found in 55\% of all possible component combinations of 2, 3, and 4 (Table 2). More synergistic effects were observed among the combinations of $2(67 \%)$ than were observed among the combinations of 3 $(50 \%)$ or $4(0 \%)$. This is the opposite of what was found in the blueberry study with individual compounds. The more complex mixtures of antioxidant compounds found in grape components, when blended, may saturate the results.

Combinations that included skin were more often synergistic. All three varieties showed synergism when both skin and juice were combined as well as skin, juice, and seed. The Red Globe skin+juice+seed was significantly more synergistic than the other two varieties. However, adding pulp to a combination nullified the synergistic effect (skin+pulp+juice, all three varieties) and caused an antagonistic effect in some cases (Sugraone skin+pulp+seed and Black Ribier skin+pulp+seed+juice). Again, this may be due to different compounds found in the respective fractions or ratio percentages, which would impact the chemical interactions.

Sandhu and Gu (2010) compared the antioxidant capacity of skin, seed, and pulp of eight different grape cultivars using ORAC. The antioxidant capacity of the phenolic compounds responsible for the antioxidant activity was highest in seed (87.1\%), then skin (11.3\%), and then pulp (1.6\%). This explains our finding that pulp did not induce synergistic activity in combination with other components due to its low phenolic content. It does not explain the antagonistic effect however.

Table 2. ORAC and TEAC differences for combinations minus the individual components in the combination (Equation 1 to Equation 3) ${ }^{1}$

\begin{tabular}{lllllll}
\hline \multicolumn{5}{c}{ ORAC $(\boldsymbol{\mu m o l ~ T E} / \mathbf{g})$} & \multicolumn{3}{l}{ TEAC(mM) } \\
\hline Combination & Black Ribier & Red Globe & Sugraone & Black Ribier & Red Globe & Sugraone \\
$\mathbf{S k} / \mathbf{P}^{2}$ & $25.5 \pm 3.5^{*}$ & $2.08 \pm 4.6$ & $16.0 \pm 2.0^{*}$ & $0.21 \pm 0.1^{*}$ & $0.23 \pm 0.06^{*}$ & $0.04 \pm 0.09$ \\
$\mathbf{S k} / \mathbf{S e}$ & $54.2 \pm 3.5^{*}$ & $19.3 \pm 4.6^{*}$ & $-10.8 \pm 2.0^{*}$ & $0.46 \pm 0.1^{*}$ & $0.73 \pm 0.06^{*}$ & $-0.06 \pm 0.09$ \\
$\mathbf{S k} / \mathbf{J}$ & $21.0 \pm 3.5^{*}$ & $39.3 \pm 4.6^{*}$ & $15.8 \pm 2.0^{*}$ & $0.09 \pm 0.1$ & $0.89 \pm 0.06^{*}$ & $0.15 \pm 0.09$ \\
$\mathbf{P} / \mathbf{S e}$ & $90.0 \pm 3.5^{*}$ & $5.32 \pm 4.6$ & $-1.74 \pm 2.0$ & $0.81 \pm 0.1^{*}$ & $0.33 \pm 0.06^{*}$ & $-0.01 \pm 0.09$ \\
$\mathbf{J} / \mathbf{S e}$ & $1.96 \pm 3.5$ & $1.50 \pm 4.6$ & $0.16 \pm 2.0$ & $-0.06 \pm 0.1$ & $-0.001 \pm 0.06$ & $0.04 \pm 0.09$ \\
$\mathbf{P} / \mathbf{J}$ & $6.06 \pm 3.5$ & $5.74 \pm 4.6$ & $1.03 \pm 2.0$ & $-0.07 \pm 0.1$ & $0.07 \pm 0.06$ & $0.01 \pm 0.09$ \\
$\mathbf{S k} / \mathbf{P} / \mathbf{S e}$ & $4.87 \pm 3.5$ & $6.92 \pm 4.6$ & $-3.96 \pm 2.0$ & $0.31 \pm 0.1^{*}$ & $0.18 \pm 0.06^{*}$ & $0.01 \pm 0.09$ \\
$\mathbf{P} / \mathbf{S e} / \mathbf{J}$ & $1.25 \pm 3.5$ & $1.35 \pm 4.6$ & $5.10 \pm 2.0^{*}$ & $-0.03 \pm 0.1$ & $-0.05 \pm 0.06$ & $0.03 \pm 0.09$ \\
$\mathbf{S k} / \mathbf{J} / \mathbf{S e}$ & $11.2 \pm \pm 3.5^{*}$ & $112 \pm 4.6^{*}$ & $6.04 \pm 2.0^{*}$ & $0.14 \pm 0.1$ & $0.98 \pm 0.06^{*}$ & $0.14 \pm 0.09$ \\
$\mathbf{S k} / \mathbf{P} / \mathbf{J}$ & $4.07 \pm 3.5$ & $0.51 \pm 4.6$ & $3.77 \pm 2.0$ & $-0.01 \pm 0.1$ & $0.003 \pm 0.06$ & $0.09 \pm 0.09$ \\
$\mathbf{S k} / \mathbf{P} / \mathbf{S e} / \mathbf{J}$ & $-0.32 \pm 3.5$ & $1.96 \pm 4.6$ & $2.50 \pm 2.0$ & $-0.16 \pm 0.1$ & $-0.04 \pm 0.06$ & $0.12 \pm 0.09$ \\
\hline
\end{tabular}

${ }^{1}$ Each value is the mean ORAC $\left(\mu \mathrm{mol}\right.$ Trolox/g) or TEAC $(\mathrm{mM})$ difference $\pm \mathrm{SE} . \mathrm{n}=3 .{ }^{2} \mathrm{Sk}=\mathrm{Skin}, \mathrm{P}=\mathrm{Pulp}$, $\mathrm{Se}=\mathrm{Seed}$, J=Juice. ${ }^{*}$ Indicates a statistically significant synergy (positive values) or antagonism (negative values) between the combination and the individual components. 


\subsubsection{TEAC}

The Black Ribier variety showed a statistically higher TEAC value for juice and Red Globe for seeds (Table 1). The other three components were not statistically different between the three varieties.

Surprisingly similar to the ORAC assay, synergy was found in $55 \%$ of all possible component combinations of 2 , 3 , and 4 . More synergistic effects were observed among the combinations of $2(67 \%)$ than were observed among the combinations of $3(50 \%)$ or $4(0 \%)$ (Table 2$)$.

\subsubsection{Correlation Between ORAC and TEAC}

Differences across assays were expected (Tabart et al., 2009). Tabart et al. suggested that the reason for this variance is that ORAC is the only method that uses inhibition time, degree of inhibition, and a completed reaction. Wang et al. (2011) observed that of the 4 assays they used to measure antioxidant activity (ORAC, DPPH, Total Phenolic Content (TPC) and Ferric Reducing Ability of Plasma (FRAP)), generally only one assay would show synergy for any given combination. Our results with grapes also aligned with this finding, with only $3 / 30$ combinations showing synergy in both ORAC and TEAC, despite the similar number of combinations demonstrating synergy across the two assays.

\subsection{Chocolate Covered Strawberries Study}

\subsubsection{ORAC Synergy at the Mixed Whole Food Level}

When extracts of each of the chocolates and strawberries were compared statistically to the extracts of the four types of chocolate covered strawberries for potential synergy (Equation 1), a significant interaction was only found in one group; the $88 \%$ cocoa chocolate fully-dipped strawberries ( $88 \mathrm{~F}$, data not shown).

\subsubsection{ORAC Synergy at the Chemical Level}

Chocolate covered strawberries were also analyzed at the chemical level (Figure 4). The ORAC $\mathrm{LP}_{\mathrm{LP}}$ values of the lipophilic extracts of both strawberries and chocolate were negligible, so only the ORAC $\mathrm{HP}_{\mathrm{HP}}$ values of the hydrophilic extracts were evaluated. All possible combinations of 2, 3, 4, and 5 of (+)-catechin, quercetin, pelargonidin, $p$-coumaric acid, (-)-epicatechin were combined at the ratios found in $88 \mathrm{~F}$ chocolate covered strawberries. Of all statistically significant combinations, $73 \%$ showed synergism. Of the combinations that were statistically significant for synergism, $81 \%$ included catechin. Catechin and $p$-coumaric acid showed a strong synergistic relationship, as found in Reber et al. (2011). Ninety five percent of all combinations that included both catechin and $p$-coumaric acid were statistically synergistic. Combinations consisting of only two compounds were more likely to exhibit synergism. It was found that of all statistically significant antagonistic combinations, $70 \%$ included quercetin.

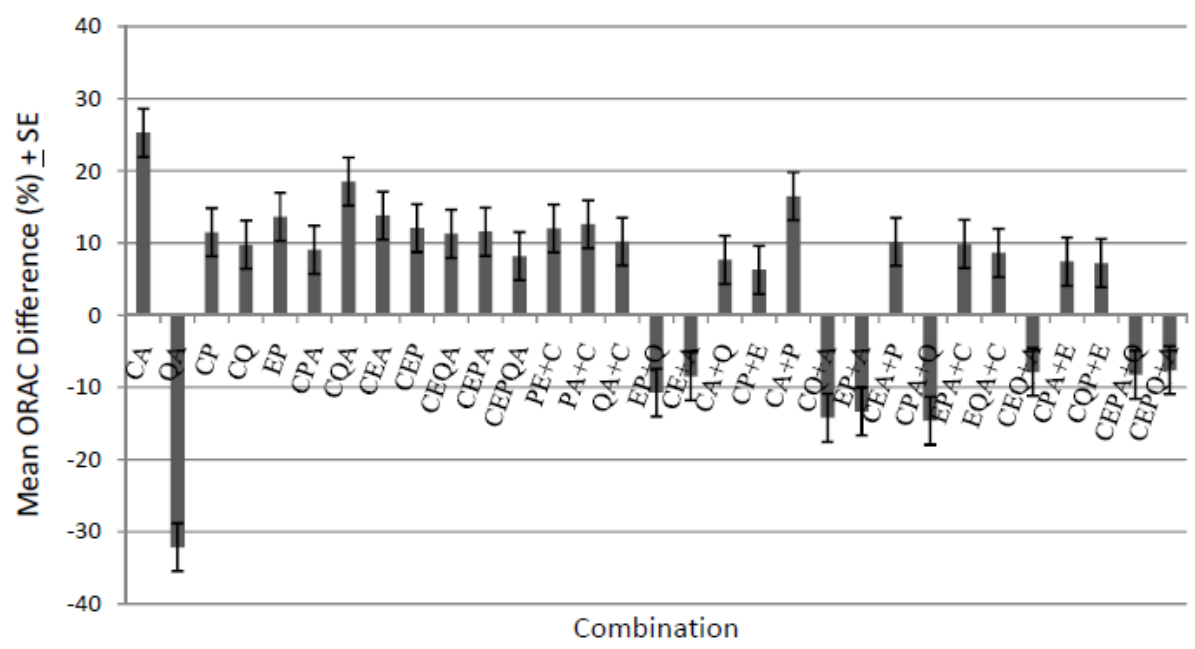

Figure 4. Oxygen Radical Absorbance Capacity (ORAC) differences \pm SE for chocolate covered strawberry chemical combinations. Values are antioxidant capacities for combinations minus the individual compounds in the combination (Equation 1 to Equation 3, no plus sign) and combinations of 3 phenolic compounds minus the sum of the $2+1$ data (Eq. 4, plus sign), $3+1$ data (Equation 5, plus sign) or $4+1$ data. Only statistically significant $(\mathrm{p}<$ $0.05)$ combinations are shown $(\mathrm{n}=6) . \mathrm{C}=(+)$-catechin, $\mathrm{Q}=$ quercetin, $\mathrm{P}=$ pelargonidin, $\mathrm{A}=p$-coumaric acid, $\mathrm{E}=$ (-)-epicatechin. The difference was then divided by the mean ORAC of the combination, giving a percentage 
Parker et al. (2010) reported that more complex combinations of antioxidants tended to show more synergy when measured by the ORAC assay. Our results in this study partially agreed with this. There were fewer combinations of 4 antioxidants that showed synergy than combinations of 2 or 3; however, combinations of 4 that did show synergy tended to have higher percentages of synergy than combinations of 3 , which were in turn higher than combinations of 2.

\subsection{Fruit Smoothies Study}

\subsubsection{ORAC}

Smoothies 1, 3, 4, and 5 were all statistically different from each other in their antioxidant capacity (Figure 5). Compared to the controls, all fruit-containing smoothies exhibited a much larger antioxidant capacity. Smoothie 3 exhibited the highest antioxidant capacity, showing blueberries increased antioxidant capacity more than raspberries or strawberries. This is consistent with the USDA Database for the Flavonoid Content of Selected Foods (USDA Database for the Flavonoid Content of Selected Foods, 2007) which showed blueberries contained a higher percentage of compounds exhibiting antioxidant capacity than either raspberries or strawberries. Hunter et al. (2012) found that milk which contained fruit and vegetable extracts had a positive effect on antioxidant markers when consumed regularly over a prolonged period compared to milk alone. This is likely due to the phytochemical content of the fruit, and is supported by our data.

Soymilk enhanced the antioxidant capacity of the smoothie. This is seen in the difference between smoothie 1 (soymilk added) and 5 (water only) (Figure 5). A possible explanation is that soymilk has many different components available to interact with the antioxidant compounds found in the fruit. Soy also contains phenolic compounds which could contribute in a positive way to the interaction. This can also be observed in control smoothie 2 vs. 3 where soymilk was removed; the antioxidant capacity decreased.

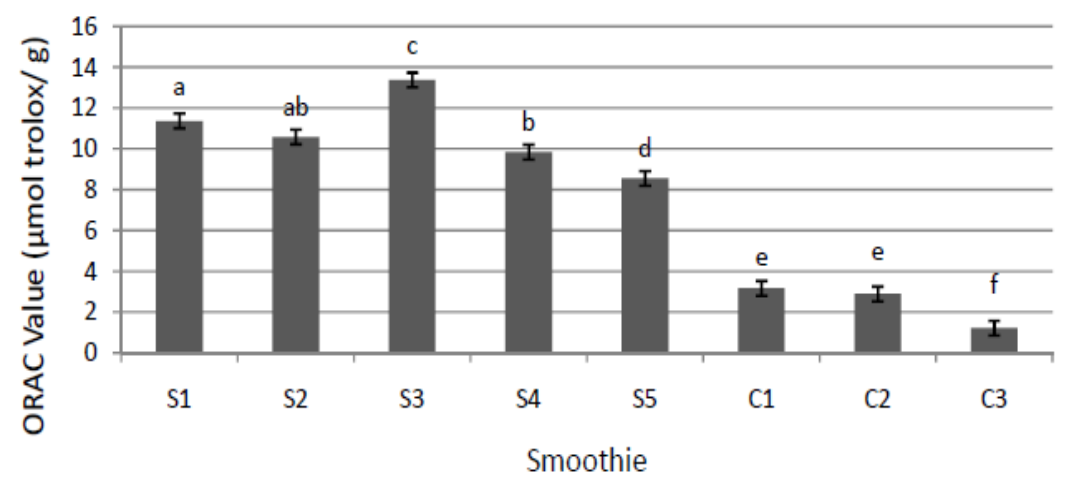

Figure 5. ORAC value $(\mu \mathrm{mol} \mathrm{TE} / \mathrm{g})$ of smoothies \pm SE. Smoothies that do not share a lowercase letter are statistically different $(n=3, p<0.05)$. The contents of the smoothies are: S1-Fruit Combination + Milk (360 ml Soymilk, $120 \mathrm{~g}$ frozen yogurt and $60 \mathrm{~g}$ ice (milk blend), $60 \mathrm{~g}$ each of blueberries, strawberries, and raspberries (fruit blend)), S2- Strawberries + Milk (milk blend and $180 \mathrm{~g}$ strawberries), S3- Blueberries + Milk (milk blend and $180 \mathrm{~g}$ blueberries), S4- Raspberries + Milk (milk blend and $180 \mathrm{~g}$ raspberries), S5- Fruit Combination + Water (360 ml dd water, $120 \mathrm{~g}$ frozen yogurt, $60 \mathrm{~g}$ ice and fruit blend), C1- Milk + Water (360 ml soymilk, 300 $\mathrm{ml} \mathrm{dd} \mathrm{water,} \mathrm{and} 60 \mathrm{~g}$ ice), C2- Milk + Frozen Yogurt + Water $(360 \mathrm{ml}$ soymilk, $120 \mathrm{~g}$ frozen yogurt, $180 \mathrm{ml} \mathrm{dd}$ water, and $60 \mathrm{~g}$ ice), C3- Water + Frozen Yogurt (120 g frozen yogurt, $540 \mathrm{ml} \mathrm{dd}$ water, and $60 \mathrm{~g}$ ice)

Antioxidant capacity was measured in all smoothie types at various time intervals after smoothie blending and the averages were calculated (data not shown). This was to determine if fruit enzymatic activity would affect antioxidant capacity over time and if it would be different in more complex exo-interactions. There was a significant difference in the antioxidant capacity between time 0 (time of creation) and $30 \mathrm{~min}$ and $45 \mathrm{~min}$, showing a general decreasing trend in antioxidant capacity as time passed. The difference from time 0 to time 45 (a change of $8.8 \%$ ) may be too small to have a practical significance in decreasing the nutritional value of a smoothie when considering the typical time it takes to consume one.

Total phenolic content decreased significantly from $0 \mathrm{~min}$ to $15 \mathrm{~min}$, but did not significantly decrease further at 30 or $45 \mathrm{~min}$ (data not shown). Enzymes liberated in the blended fruit may have changed the phenolic 
composition of the fruit more initially, and then slowed. However the magnitude of the change from time 0 to 15 was small despite its significance.

The $\mathrm{L}^{*} \mathrm{a} * \mathrm{~b}$ color analysis was used to determine changes in color of the smoothies over time (data not shown). Overall, as time passed the smoothies became lighter (statistically different only between times 0 and 15), less red (statistically different at each time interval from time zero), and less blue (statistically different between time 0 and times 30 and 45). Visually, the difference was not noticeable. It may have been due to the ice melting and changing the refractance, as total phenolic content did not change after $15 \mathrm{~min}$. Or, the phenolic changes resulted in the formation of other phenolic compounds which affected the color, but not the total phenolic content.

\section{Limitations}

Only a limited number of phenolic compounds could be studied in the chemical analysis in the blueberry and chocolate covered strawberries studies. Thus our results do not fully represent the whole food. Only a small sampling of grapes, chocolate and strawberries was purchased, so the results may not extrapolate to common consumption. The differences between mechanisms of the assays used will affect the measurement of the interactions (Tabart et al., 2009). Synergistic combinations of phytochemicals found using one assay did not strongly predict results found using a different assay.

\section{Conclusions}

\subsection{Blueberry Study}

When measured by ORAC, combinations that included both catechin and malvidin were more than twice as synergistic as other combinations, suggesting they play important roles in determining the synergy in a combination. There was a positive correlation between the number of compounds in a mixture and its $\%$ synergy. There was little correlation across the ORAC, TEAC, and DPPH assays when measuring the synergy of antioxidant combinations.

\subsection{Grape Study}

Across three grape cultivars (Black Ribier, Red Globe, and Sugraone), the Black Ribier cultivar exhibited the highest whole grape antioxidant capacity. Combinations of components including the skin were more likely to be synergistic. Both the TEAC and ORAC assays resulted in synergy in $55 \%$ of all possible combinations of 2,3 , and 4.

\subsection{Chocolate Covered Strawberries Study}

There was only one synergistic result when looking at synergy at the mixed whole food level $(88 \%$ cocoa chocolate fully dipped). At the chemical level, catechin and $p$-coumaric acid had the most synergistic relationship and were the most important contributors to antioxidant synergy when both were part of other combinations.

\subsection{Fruit Smoothies Study}

Fruit smoothies made with blueberries demonstrated higher antioxidant capacity than those made with strawberries or raspberries, or a combination of all three. Also, soymilk showed an apparent synergistic relationship with fruit as compared to water. Overall, as time passed after blending, the smoothies became lighter, less red, and less blue, though with little phenolic change.

\subsection{Overall}

Little correlation was found among the varying assays in the blueberry, grape, and chocolate covered strawberries studies. In the blueberry and chocolate covered strawberries studies, catechin was present in a majority of synergistic mixtures. In the grape and fruit smoothies studies, the fruit or variety with the darkest skin color exhibited the highest antioxidant capacity (Black Ribier grapes, and blueberries) and skin was present or contributed strongly to apparent synergy. Overall, the complexity of a mixture, whether an endo- or exo-interaction, appears to be the most important factor in the antioxidant capacity of various chemical mixtures, food components or whole foods. Further work is needed to better understand the precise ratios and concentrations that are the most effective producers of synergy in these and other foods.

\section{Acknowledgements}

The Brigham Young University Department of Nutrition, Dietetics and Food Science provided the funding for these studies. 


\section{References}

Awika, J. M., Rooney, L. W., Wu, X. L., Prior, R. L., \& Cisneros-Zevallos, L. (2003). Screening methods to measure antioxidant activity of sorghum (Sorghum bicolor) and sorghum products. J. Agric. Food Chem., 51, 6657-6662. http://dx.doi.org/10.1021/jf034790i

Chu, Y. F., Sun, J., Wu, X., \& Liu, R. H. (2002a). Antioxidant and antiproliferative activities of common fruits. $J$. Agric. Food Chem., 50, 7449-7454. http://dx.doi.org/10.1021/jf0207530

Chu, Y. F., Sun, J., Wu, X., \& Liu, R. H. (2002b). Antioxidant and antiproliferative activities of common vegetables. J. Agric. Food Chem., 50, 6910-6916. http://dx.doi.org/10.1021/jf020665f

Crozier, A., Clifford, M. N., \& Ashihara, H. (Eds.) (2006). Plant Secondary Metabolites. Oxford: Blackwell Publishing. http://dx.doi.org/10.1002/9780470988558

Davalos, A., Gomez-Cordoves, C., \& Bartolome, B. (2004). Extending applicability of the oxygen radical absorbance capacity (ORAC-Fluorescein) assay. $J$ Agric Food Chem., 52, 48-54. http://dx.doi.org/10.1021/jf0305231

Eberhardt, M. V., Lee, C. Y., \& Liu, R. H. (2000). Nutrition-antioxidant activity of fresh apples. Nature, 405, 903-904. http://dx.doi.org/10.1038/35016151

Franke, A., Custer, L., Arakaki, C., \& Murphy, S. (2004). Vitamin C and flavonoid levels of fruits and vegetables consumed in Hawaii. J. Food Composit Anal., 17, 1-35. http://dx.doi.org/10.1016/S0889-1575(03)00066-8

Freeman, B. L., Eggett, D. L., \& Parker, T. L. (2010). Synergistic and antagonistic interactions of phenolic compounds found in navel oranges. J. Food Sci., 75, 570-576. http://dx.doi.org/10.1111/j.1750-3841.2010.01717.x

Gao, Y., \& Cahoon, G. A. (1994). Cluster shading effects on fruit-quality, fruit skin color, and anthocyanin content and composition in reliance (Vitis hybrid). VITIS, 33, 205-9.

Harnly, J. M., Doherty, R. F., Beecher, G. R., Holden, J. M., Haytowitz, D. B., Bhagwat, S., \& Gebhardt, S. (2006). Flavonoid content of U.S. fruits, vegetables, and nuts. J. Agric. Food Chem., 54, 9966-9977. http://dx.doi.org/10.1021/jf061478a

Hidalgo, M., De La Rosa, C., Carrasco, H., Cardona, W., \& Gallardo, C. (2009). Antioxidant capacity of eugenol derivatives. Quim. Nova, 32, 1467-70. http://dx.doi.org/10.1590/S0100-40422009000600020

Hidalgo, M., Sanchez-Moreno, C., \& de Pascual-Teresa, S. (2010). Flavonoid-flavonoid interaction and its effect on their antioxidant activity. J. Food Chem., 121, 691-696. http://dx.doi.org/10.1016/j.foodchem.2009.12.097

Hunter, D. C., Brown, R., Green, T., Thomson, C., Skeaff, M., Williams, S., ..., Skinner, M. A. (2012). Changes in markers of inflammation, antioxidant capacity and oxidative stress in smokers following consumption of milk, and milk supplemented with fruits and vegetables. Int J Food Sci Nutri., 63, 90-102. http://dx.doi.org/10.3109/09637486.2011.601286

Hwang, J., Hodis, H. N., \& Sevanian, A. (2001). Soy and alfalfa phytoestrogen extracts become potentlow-density lipoprotein antioxidants in the presence of acerola cherry extract. J. Agric. Food Chem., 49, 308-314. http://dx.doi.org/10.1021/jf0007028

Kayano, S., Kikuzaki, H., Fukutsuka, N., Mitani, T., \& Nakatani, N. (2002). Antioxidant activity of prune (Prunus domestica L.) constituents and a new synergist. J. Agric. Food Chem., 50(13), 3708-3712. http://dx.doi.org/10.1021/jf0200164

Liao, K., \& Yin, M. (2000). Individual and combined antioxidant effects of seven phenolic agents in human erythrocyte membrane ghosts and phosphatidylcholine liposome systems: importance of the partition coefficient. J. Agric Food Chem., 48, 2266-2270. http://dx.doi.org/10.1021/j9990946w

Lila, M. A., \& Raskin, I. (2005). Health-related interactions of phytochemicals. J. Food Sci., 70, R20-27. http://dx.doi.org/10.1111/j.1365-2621.2005.tb09054.x

Lutz, M., Jorquera, K., Cancino, B., Ruby, R., \& Henriquez, C. (2011). Phenolics and antioxidant capacity of table grape (Vitis vinifera L.) cultivars grown in Chile. $J$ Food Sci., 67, C1088-93. http://dx.doi.org/10.1111/j.1750-3841.2011.02298.x

Miller, N. J., \& Rice-Evans, C. A. (1997). The relative contributions of ascorbic acid and phenolic antioxidants to the total antioxidant activity of orange and apple fruit juices and blackcurrant drink. J. Food Chem., 60, 


\section{1-7. http://dx.doi.org/10.1016/S0308-8146(96)00339-1}

Parker, T. L., Miller, S. A., Myers, L. E., Migues, F. E., \& Engeseth, N. J. (2010). Evaluation of synergistic antioxidant potential of complex mixtures using oxygen radical absorbance capacity (ORAC) and electron paramagnetic resonance (EPR). J. Agric. Food Chem., 58(1), 209-217. http://dx.doi.org/10.1021/jf903080f

Prior, R. L., Hoang, H., Gu, L., Wu, X., Bacchiocca, M., Howard, L., ..., Jacob, R. (2003). Assays for hydrophilic and lipophilic antioxidant capacity (oxygen radical absorbance capacity $\left(\mathrm{ORAC}_{\mathrm{FL}}\right)$ ) of plasma and other biological and food samples. J. Agric. Food Chem., 51, 3273-3279. http://dx.doi.org/10.1021/jf0262256

Re, R., Pellegrini, N., Proteggente, A., Pannala, A., Yang, M., \& Rice-Evans, C. (1999). Antioxidant activity applying an improved ABTS radical cation decolorization assay. J. Free Radical Biol. Med., 26, 1231-1237. http://dx.doi.org/10.1016/S0891-5849(98)00315-3

Reber, J. D., Eggett, D. L., \& Parker, T. L. (2011). Antioxidant capacity interactions and a chemical/structural model of phenolic compounds found in strawberries. Int. J. Food Sci. Nutr., 62(5), 445-452. http://dx.doi.org/10.3109/09637486.2010.549115

Robards, K., Prenzler, P. D., Tucker, G., Swatsitang, P., \& Glover, W. (1999). Phenolic compounds and their role in oxidative processes in fruits. J. Food Chem., 66, 401-436. http://dx.doi.org/10.1016/S0308-8146(99)00093-X

Sandhu, A. K., \& Gu, L. (2010). Antioxidant capacity, phenolic content, and profiling of phenolic compounds in the seeds, skin, and pulp of Vitis rotundifolia (Muscadine grapes) as determined by HPLC-DAD-ESI-MS(n). J. Agric. Food Chem., 58, 4681-92. http://dx.doi.org/10.1021/jf904211q

Seeram, N. P., Adams, L. S., Hardy, M. L., \& Heber, D. (2004). Total cranberry extract versus its phytochemical constituents: Antiproliferative and synergistic effects against human tumor cell lines. J. Agric. Food Chem., 52, 2512-2517. http://dx.doi.org/10.1021/jf0352778

Serafini, M., Maiani, G., \& Ferro-Luzzi, A. (1998). Alcohol-free red wine enhances plasma antioxidant capacity in humans. J. Nutr., 128, 1003-1007.

Snyder, S. M., Reber, J. D., Freeman, B. L., Orgad, K., Eggett, D. L., \& Parker, T. L. (2011). Controlling for sugar and ascorbic acid, a mixture of flavonoids matching navel oranges significantly increases human postprandial serum antioxidant capacity. J. Nutr. Res., 31, 519-526. http://dx.doi.org/10.1016/j.nutres.2011.06.006

Steinmetz, K. A., \& Potter, J. D. (1996). Vegetables, fruit, and cancer prevention: A review. JADA, 96, 1027-39. http://dx.doi.org/10.1016/S0002-8223(96)00273-8

Su, J. D., Osawa, T., Kawakishi, S., \& Namiki, M. (1987). A novel antioxidative synergist isolated from Osbeckia chinensis L. J. Agric. Biol. Chem., 51, 3449-3450. http://dx.doi.org/10.1271/bbb1961.51.3449

Swain, T., \& Hillis, E. (1959). The phenolic constituents of Prunus domestica. The quantitative analysis of phenolic constituents. J. Sci. Food Agric., 10, 63-68. http://dx.doi.org/10.1002/jsfa.2740100110

Tabart, J., Kevers, C., Pincemail, J., Defraigne, J. O., \& Dommes, J. (2009). Comparative antioxidant capacities of phenolic compounds measured by various tests. J. Food Chem., 113(4), 1226-1233. http://dx.doi.org/10.1016/j.foodchem.2008.08.013

USDA Database for the Flavonoid Content of Selected Foods [Internet]. Release 2.1. Beltsville, MD: U.S. Department of Agriculture; c2007. Available from: http://www.ars.usda.gov/nutrientdata. Accessed May 3, 2012.

Van’t Veer, P., Jansen, M. C., Klerk, M., \& Kok, F. J. (2000). Fruits and vegetables in the prevention of cancer and cardiovascular disease. J. Public Health Nutr., 3, 103-7.

Wang, C. Y., Chen, C., \& Wang, S. Y. (2009). Changes of flavonoid content and antioxidant capacity in blueberries after illumination with UV-C. J. Food Chem., 117, 426-431. http://dx.doi.org/10.1016/j.foodchem.2009.04.037

Wang, S., Meckling, K. A., Marcone, M. F., Kakuda, Y., \& Tsao, R. (2011). Synergistic, additive, and antagonistic effects of food mixtures on total antioxidant capacities. J. Agric. Food Chem., 59, 960-968. http://dx.doi.org/10.1021/jf1040977

Wu, X., Beecher, G. R., Holden, J. M., Haytowitz, D. B., Gebhardt, S. E., \& Prior, R. L. (2004). Lipophilic and 
hydrophilic antioxidant capacities of common foods in the United States. J. Agric. Food Chem., 52, 4026-4037. http://dx.doi.org/10.1021/jf049696w

Yang, J., \& Liu, R. H. (2009). Synergistic effect of apple extracts and quercetin 3- $\beta$-D-glucoside combination on antiproliferative activity in MCF-7 human breast cancer cells in vitro. J. Agric. Food Chem., 57, 8581-8586. http://dx.doi.org/10.1021/jf8039796

Zheng, W., \& Wang, S. Y. (2003). Oxygen radical absorbing capacity of phenolics in blueberries, cranberries, chokeberries, and lingonberries. J. Agric. Food Chem., 51, 502-9. http://dx.doi.org/10.1021/jf020728u

\section{Copyrights}

Copyright for this article is retained by the author(s), with first publication rights granted to the journal.

This is an open-access article distributed under the terms and conditions of the Creative Commons Attribution license (http://creativecommons.org/licenses/by/3.0/). 